\title{
152 ADVANCE CARE PLANNING (ACP) WITHIN A PALLIATIVE APPROACH FOR RESIDENTIAL AGED CARE
}

10.1136/bmjspcare-2011-000053.152

R Hudson Respecting Patient Choices, Austin Health, Melbourne, Australia

In a unique collaboration between palliative care and aged care the Australian government produced Guidelines for a palliative approach in residential aged care, endorsed by the National Health \& Medical Research Council in 2006. Specific guidelines for Advance Care Planning (ACP) fit within the broader palliative approach with its emphasis on holistic assessment early in the course of life-threatening disease. Replacing ad hoc arrangements for offering end-of-life choices, the guidelines emphasise systematic implementation, regular education and ongoing assessment. The ACP component is clearly linked to many of the other 76 guidelines, demonstrating the role of ACP in the broader framework of goal setting and care planning.

Two brief, contrasting case studies exemplify the successful application of the guidelines for (a) a very physically frail, mentally alert older person and (b) a person with advanced dementia and other co-morbidities. Noting the families' reports and the endorsement of professional colleagues, aged care workers enjoyed pride and satisfaction from seeing the guidelines at work. The challenge remains, however, to ensure consistency in the application of the guidelines over a sustained period of time.

When advance care planning in care homes is based on endorsed guidelines drawn from the best available evidence, the end-of-life experience for older persons, families, and care staff can be transformed. 